\title{
Periodicity of mass extinctions without an extraterrestrial cause
}

\author{
Adam Lipowski ${ }^{1}$ \\ ${ }^{1}$ Faculty of Physics, Adam Mickiewicz University, 61-614 Poznań, Poland
}

\begin{abstract}
We study a lattice model of a multi-species prey-predator system. Numerical results show that for a small mutation rate the model develops irregular long-period oscillatory behavior with sizeable changes in a number of species. The periodicity of extinctions on Earth was suggested by Raup and Sepkoski but so far is lacking a satisfactory explanation. Our model indicates that this might be a natural consequence of the ecosystem dynamics, not the result of any extraterrestrial cause.
\end{abstract}

The Earth ecosystem is certainly a subject of intensive multidisciplinary research. Researchers in this field believe that at least some basic understanding of this immensely complex system can be obtained using relatively simple models, that nevertheless grasp some aspects of its rich behavior [1]. Of particular interest in physicists community is the dynamics of extinctions of species 2]. Palaeontological data, that show broad distributions of these events in the Earth history, suggest existence of strong, perhaps power-law correlations between extinctions. Similar correlations appear in the so-called critical systems and such an analogy resulted in a wealth of interesting models that consider extinctions as a natural consequence of the dynamics of an ecosystem [3]. However, fossil data are not entirely convincing, and it is not clear to what extent the analogy with critical systems hold. What is more, a number of researchers prefer an alternative explanation where extinctions appear due to external stresses imposed on the ecosystem as, e.g., impacts of comets or meteorites, or an increased volcanic activity [2]. The popularity of theories of exogenous origin of extinctions increased when Raup and Sepkoski concluded from analyzing fossil date that big extinction events during the last $250 \mathrm{My}$ (million years) have been occurring with periodicity of about $26 \mathrm{My}$ [4]. Several theories, mostly of astronomical origin, have been proposed to explain such a periodicity, but none of them is confirmed or commonly accepted [5]. Although the Raup and Sepkoski analysis was put into question [6], the more recent analysis confirms a similar periodicity of extinctions [7] keeping this fascinating hypothesis still open.

Lacking a firm evidence of any exogenous cause, one can ask whether the periodicity of extinctions can be explained without referring to such a factor. Or in other words, if it is possible that the ecosystem dynamics produces (by itself) oscillations on such a long time scale. Since the seminal work of Lotka and Volterra, an oscillatory behavior is already well-known in various preypredator systems $[1,8]$, but the periodicity of oscillations of densities in such systems, that is determined by the growth and death rate coefficients of interacting species, is of the order of a few years rather than millions. Preypredator systems, where such an oscillatory behavior was studied, are typically quite simple and consist of a fixed and rather small number of species. Certainly a model capable of describing the dynamics of extinctions should include a large number of species as well as mutation and competition mechanisms. There is already a wealth of papers where various models of this kind where examined [9], but none of them has been reporting a longterm periodicity of extinctions. There is, however, one aspect that these models are missing and that is perhaps quite important, namely they neglect spatial correlations between organisms. From statistical mechanics we already know that when the spatial dimension of the embedding space is rather low, such correlations might play an important role, and hence more realistic models of the ecosystem should take them into account.

In the present paper we study a multi-species lattice model of an ecosystem. In our model predator species compete for food (prey) and space (to place an offspring). This competition combined with a mutation mechanism leads to the periodic behavior, although some characteristics of our model, as, e.g., the number of species, show in addition strong stochastic irregularities. Sometimes our system is populated by a group of medium-efficiency species. But this coexistence at a certain moment is interrupted by creation of a species that is more efficient and able to invade even a substantial part of the system. However, the reign of such an apex predator does not last long. It is a fast-consuming species and it quickly decimates the population of preys, which in turn leads to its own decline. Such a situation opens up niches that again become occupied by less-effective species that survived the invasion or were created by mutation, and the situation repeats. Simulations show that the smaller the mutation probability, the larger the periodicity of such a behavior. Although it is difficult to access, we expect that the mutation rate in real ecosystems, as interpreted in the context of our model [10], is very small and the presented model might at least suggest an explanation of the $26 \mathrm{My}$ periodicity of big extinctions as a natural consequence of the ecosystem dynamics, not as the result of an external perturbation.

Our model is a multi-species extension of an already examined prey-predator model [1]. At each site $i$ of a square lattice of linear size $N$ we have the four-state operator $x_{i}$ that corresponds to this site being empty $\left(x_{i}=0\right)$, occupied by a prey $\left(x_{i}=1\right)$, by a predator $\left(x_{i}=2\right)$, or by both of them $\left(x_{i}=3\right)$. Each preda- 
tor is characterized by a real number parameter $m_{i}$ $\left(0<m_{i}<1\right)$ that we will call size $\left(m_{i}\right.$ is meaningful only when $i$ is occupied by a predator). We also introduce the relative update rate of preys and predators $r(0<r<1)$, and the mutation probability $p$. The dynamics of this model is specified as follows:

(a) Choose a site at random (the chosen site will be denoted by $i$ ).

(b) With the probability $r$ update a prey at site $i$ (i.e., if $x_{1}=1$ or $x_{i}=3$, otherwise do nothing). Provided that at least one neighbor ( say $j$ ) of the chosen site is not occupied by a prey (i.e., $x_{j}=0$ or $x_{j}=2$ ), the prey at the site $i$ produces an offspring and places it on an empty neighboring site (if there are more empty sites, one of them is chosen randomly). Otherwise (i.e., if there are no empty sites) the prey does not breed.

(c) Provided that $i$ is occupied by a predator (i.e., $x_{1}=2$ or $x_{i}=3$ ) update this site with the probability $(1-r) m_{i}$, where $m_{i}$ is the size of the predator at site $i$. If a chosen site is occupied by a predator only $\left(x_{i}=2\right)$, it dies, i.e., the site becomes empty $\left(x_{i}=0\right)$. If there is also a prey there $\left(x_{i}=3\right)$ the predator consumes the prey (i.e., $x_{i}$ is set to 2 ) and if possible it places an offspring at an empty neighboring site. For a predator of size $m_{i}$ it is possible to place an offspring at a site $j$ provided that $j$ is not occupied by a predator $\left(x_{j}=0\right.$ or $\left.x_{j}=1\right)$ or is occupied by a predator $\left(x_{j}=2\right.$ or $x_{j}=3$ ) but of a smaller size than $m_{i}$ (in such a case a smaller-size predator is replaced by an offspring of a larger-size predator). An offspring inherits parent's size with the probability $1-p$ and with the probability $p$ it gets a new size that is drawn from a uniform distribution.

One can see that the size $m_{i}$ of a predator determines both its update rate and its strength when it competes with other predators for space. While the increased strength is always favorable, the larger update rate might be a disadvantage when preys do not reproduce fast enough. As it will be shown below, the behavior of our model is very much influenced by this property of the dynamics.

The already studied single-predator version 11 is obtained when all predators have a unit size $m_{i}=1$ and suppressed mutations $p=0$. In such a case, for $r>0.11$ the model is in an active phase with positive densities of preys $\rho_{0}$ (which is a fraction of all sites $i$ such that $x_{i}=1$ or $x_{i}=3$ ) and predators $\rho$ (fraction of all sites $i$ such that $x_{i}=2$ or $\left.x_{i}=3\right)$. For $r<0.11$ the update rate of preys is too small to sustain an active phase but it is a population of predators that becomes extinct and the model enters an absorbing state where all sites are occupied by preys. In the active phase but close to the transition point $(0.11)$ one observes oscillations of $\rho_{0}$ and $\rho$ but the amplitude of these oscillations diminishes in the thermodynamic limit $N \rightarrow \infty$. On the other hand, for the model on the three-dimensional lattice such oscil-
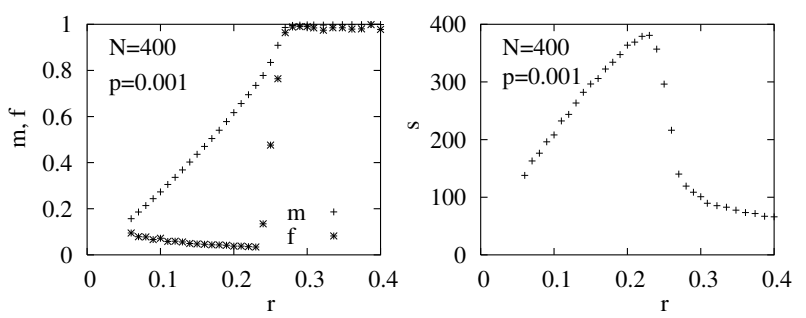

FIG. 1: Average size $m$, fraction of a dominant predator species $f$ and the number of species $s$ as a function of update rate $r$. Results of simulations do not depend on an initial configuration and usually it was a random distribution of preys and predators.

lations most likely persist in this limit [1].

To examine the behavior of our model we used simulations and measured its various characteristics such as densities of preys $\rho_{0}$ and predators $\rho$, the average size of dominant predator $f$, the average size $m$, the number of species $s$, and the lifetime of predator species. To define $s$ we classify predators into species according to their size. Some of these quantities are presented in Fig. 1] One can see that for $r>r_{c} \sim 0.27$ predators in the system belong essentially to one dominant $(f \sim 1)$ species of a large size $(m \sim 1)$. Of course, mutations create from time to time some other species but they occupy a negligible portion of a system - unless a newly created species will have a larger size than the dominant species and will be able to invade the system. Fig. 11 also shows that a much different behavior appears for $r<r_{c}$. In this case a dominant species occupies only a small fraction of a system (the comparison with the results for system size $N=200$ shows a strong $N$-dependence and suggests that for larger $N$ the fraction $f$ will diminish to zero). Moreover, the average size $m$ differs substantially from unity that indicates that having a large size is no longer advantageous feature. Another indication of a more complex behavior in this case is a large increase of the number of species.

In our opinion, it is the regime for $r<r_{c}$ whose complex dynamics might resemble the behavior of realistic ecosystems. To have a better understanding of the behavior of the model in this regime we present a time dependence of some of its characteristics. The unit of time is defined as a single, on average, update of each site (i.e., it is made of $N^{2}$ elementary single-site updates). While in Fig. 2 densities $\rho_{0}, \rho$, and the average size $m$ show a relatively regular oscillations, the number of species $s$ is much more irregular. During periods of multi-species coexistence, predators have a rather small size (they eat slowly) that enables them to sustain their density $\rho$ relatively large. As a result density of preys $\rho_{0}$ is rather small. At certain moment, however, a predator of a large size is created and starts to invade the system. As a 


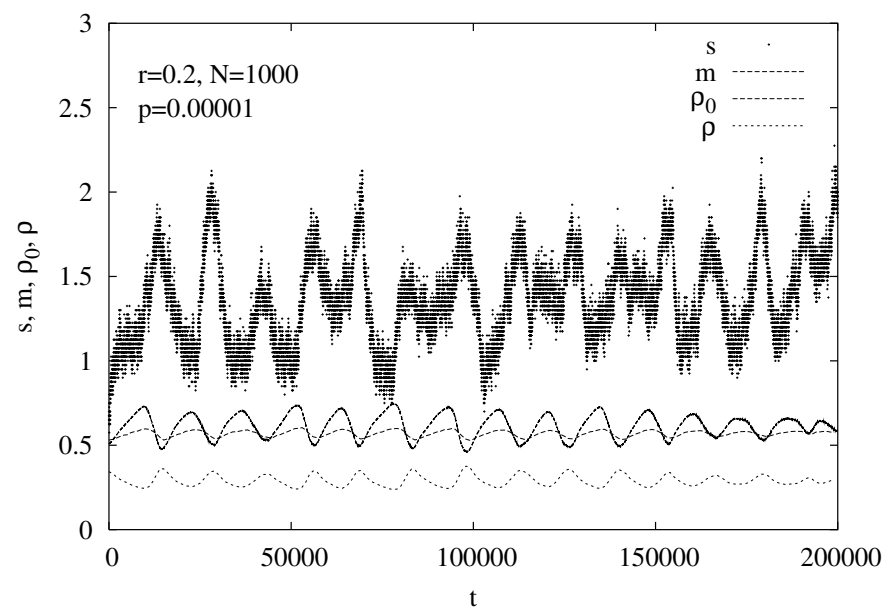

FIG. 2: Time dependence of the number of species $s$ (to superpose with other data it was divided by 40), average size $m$, density of preys $\rho_{0}$, and density of predators $\rho$.

result the number of species $s$ rapidly decreases while $m$ increases. Moreover, the density $\rho$ decreases and this is related with the fact that a predator of a large size consumes preys too quickly and is simply running out of food. Hence, the population of this predator in some places disappears and that creates areas where preys can breed without being consumed by predators and that is why the density $\rho_{0}$ after an initial short decline increases to a relatively large value. However, a large-size species cannot keep its dominance for a long time since large empty places occupied mainly by preys constitute ideal niches for other predators as well. As a result, the model is driven again toward a multispecies coexistence.

An important question is how these oscillations behave for an increasing system size $N$. Comparing (not presented) results for different values of $N$, we expect that the amplitude of these oscillations will diminish to zero (period of oscillations does not seem to depend on $N)$. This is because for a sufficiently large $N$ the system is essentially decomposed into several independent domains where multi-species and fewer-species periods are uncorrelated and fluctuations cancel out. However, there is an additional factor that is responsible for the size of these independent domains and thus the amplitude of oscillations, namely the mutation probability $p$. Indeed, the end of the multi-species period in a certain domain is induced by the creation of a large-size predator. For the decreasing mutation probability $p$ such events will be less and less frequent and multi-species domains will have more time to grow. We thus expect that for decreasing $p$ the size of such domains should increase and, as a result, for finite $N$ the amplitude of oscillations should also increase. Moreover, the period of these oscillations, that is determined by the time needed for such domains to grow, should also increase. Simulations, as shown in

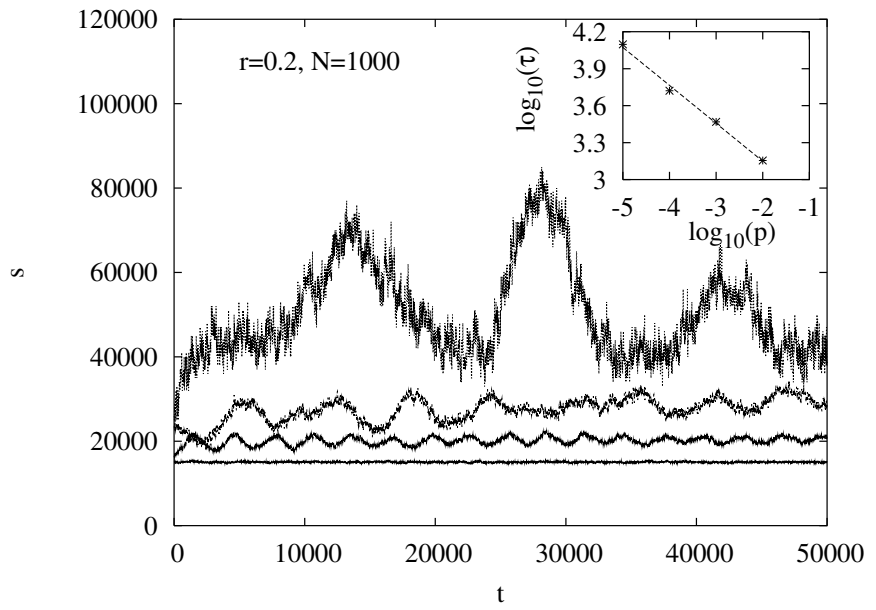

FIG. 3: The time dependence of the number of species for (from top) $p=0.00001,0.0001,0.001$, and 0.01. To superpose the data on a graph the actual values of $s$ were divided by some factors. Such an operation does not change a characteristic period of fluctuations and their relative amplitude. Inset shows the period of oscillation $\tau$ as a function of mutation probability $p$ obtained from the maximum of the Fourier transform of the time dependence of the number of species $(N=1000)$

Fig. 3. confirm such a behavior. Let us notice large fluctuations for $p=0.00001$, where the number of species after an invasion drops roughly by a factor of two. To examine the $p$-dependence of the period of oscillations $\tau$ more quantitatively, we calculated the Fourier transform of the time dependent number of species $s$ (other characteristics like $m, \rho_{0}$, or $\rho$ give basically the same result). The period of oscillations $\tau$ as extracted from the maximum of this transform is shown on the logarithmic scale in the inset of Fig. [3 Straight line fit corresponds to the dependence $\tau \sim p^{-0.31}$ but calculations for larger system size $N$ or smaller $p$ might modify this estimation.

As we already mentioned, the amplitude of oscillations in our model is determined by the combination of two factors: system size $N$ and mutation probability $p$. That $\tau$ increases for decreasing $p$ is an important result. It shows that for a small mutation probability $p$ and finite but large system size $N$ (i.e., specifications of the real ecosystem) the model develops long-period oscillatory behavior with sizeable changes of e.g., the number of species $s$. It might be interesting to notice that for the single-predator version [11], with $m_{i}=1$ and $p=0$, the period of oscillations in the two-dimensional case for $r=0.2$ is around 30. For the present model and for $p=0.00001$ the period of oscillations is larger by almost three orders of magnitude (see the inset in Fig. 3). It shows that the periodic behavior in our model has a much different mechanism than the Lotka-Volterra oscillations in simple prey-predator systems.

One of the properties often analyzed in models 


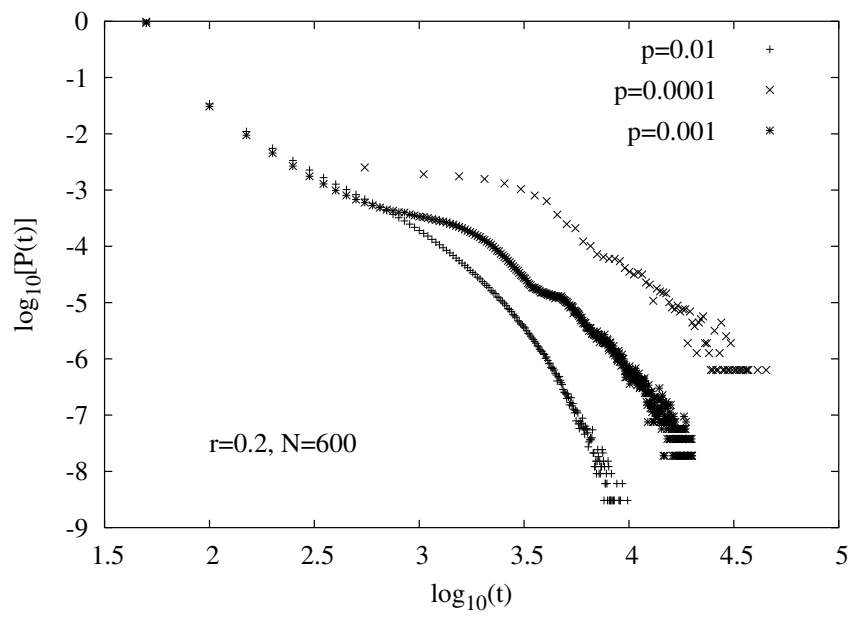

FIG. 4: Logarithmic plot of the probability distribution of lifetimes of predator species.

of ecosystems is the lifetime distribution of species. Palaeontological data suggest some broad distributions but they are again not very conclusive and both exponential and power-law fits can be made [2]. The lifetime distribution for our model is shown in Fig. 4. Although for $p=0.01$ the numerical results suggest an exponential distribution, for smaller $p$ the situation is less clear. Especially, for $p=0.00001$ it seems that a broader, perhaps a power-law distribution might better describe the lifetime of our species.

It would be interesting to make further analysis of our model. For example, one can implement a less abrupt mutation mechanism, where a new species will be only a small mutation of a parental species. Such a modification probably results in a longer period of oscillations and might be more suitable for comparison with the real ecosystem. Another possibility might be to examine the differences in, e.g., lifetime distribution of species before and after an extinction. That such differences exist is suggested by the asymmetry of our data in Fig. 2 where the changes in a pre-extinction period seem to be different than in the post-extinction one. Palaeontological data also show certain differences in longevity of species during such periods [12], and a comparison with the predictions of our model, if feasible, would be very desirable.

Acknowledgements: I thank M. Droz (Univ. of Geneva) and A. Pȩkalski (Univ. of Wrocław) for interesting discussions. The research grant 1 P03B 01427 from KBN is gratefully acknowledged.
[1] J. D. Murray, Mathematical Biology, (Springer, 1989). J. Hofbauer and K. Sigmund The Theory of Evolution and Dynamical Systems, (Cambridge University Press, 1988).

[2] M. E. J. Newman and R. G. Palmer, Modelling Extinction, (Oxford University Press, New York, 2003). M. E. J. Newman and R. G. O. Palmer, e-print: adap-org/9908002

[3] P. Bak and K. Sneppen, Phys. Rev. Lett. 71, 4083 (1993). R. V. Solé and S. C. Manrubia, Phys. Rev. E 54, R42 (1996). L. A. N. Amaral and M. Meyer, Phys. Rev. Lett. 82, 652 (1999). B. Drossel, Adv. Phys. 50, 209 (2001).

[4] D. M. Raup and J. J. Sepkoski, Proc. Natl. Acad. Sci. 81, 801 (1984).

[5] M. Davis, P. Hut, and R.A. Muller, Nature 308, 715 (1984). M. R. Rampino and R. B. Stothers, Nature 308, 709 (1984). D. P. Whitmire and A. A. Jackson, Nature 308, 713 (1984).

[6] C. Patterson and A. B. Smith, Nature 330, 248 (1987). S. M. Stanley, Paleobiology 16, 401 (1990).

[7] A. Prokoph, A. D. Fowler, and R. T. Patterson, Geology 28, 867 (2000).

[8] Various kinds of oscillatory behaviour without a periodic driving are known in for example epidemic spreading (M. Kuperman and G. Abramson, Phys. Rev. Lett. 86, 2909 (2001)) or voter-like models (A. Szolnoki and G. Szabó, Phys. Rev. E 70, 037102 (2004)).

[9] P. A. Rikvold and R. K. P. Zia, Phys. Rev. E 68, 031913 (2003). D. Chowdhury, D. Stauffer, and A. Kunwar, Phys. Rev. Lett. 90, 068101 (2003). B. Drossel and A. J. McKane, Handbook of Graphs and Networks: From the Genome to the Internet, $\mathrm{S}$. Bornholdt and H.G. Schuster (Eds) (Wiley-VCM, Berlin, 2002), e-print: nlin.AO/0202034 M. Hall, K. Christensen, S. A. di Collobiano, and H. J. Jensen, Phys. Rev. E 66, 011904 (2002). C. Quince, P. G. Higgs, and A. J. McKane, in Biological Evolution and Statistical Physics, eds. M. Lässig and A. Vallerian (Springer Verlag, Berlin-Heidelberg 2002). F. Coppex, M. Droz, and A. Lipowski, Phys. Rev. E 69, 061901 (2004).

[10] Although mutations are relatively frequent, for comparison with our model we would have to consider events that from a given species create a species that is 'substantially' different. Such events usually result as a cumulative effect of many mutations and their probability is certainly very small.

[11] A. Lipowski, Phys. Rev. E 60, 5179 (1999). A. Lipowski and D. Lipowska, Physica A 276, 456 (2000).

[12] A. I. Miller and M. Foote, Science 302, 1030 (2003). 